\title{
A Case Report of Relapsing Remitting Multiple Sclerosis: Getting the Diagnosis Sooner
}

\section{Fuentes S*}

Department of Family Medicine, Texas A\&M College of Medicine, Baylor Scott and White Medical Center, TX, USA

*Correspondling author: Fuentes S, Department of Family Medicine, Texas A \& M College of Medicine, Baylor Scott and White Medical Center, 425 University Blvd, Round Rock, TX, USA

Received: January 13, 2021; Accepted: February 27, 2021; Published: March 06, 2021

\begin{abstract}
Multiple sclerosis can often present with nonspecific symptoms leading to difficulty in establishing a diagnosis early in the disease process. Early diagnosis and treatment is of importance due to the associated decrease in disability for those that get treatment sooner. Here we present a case with a patient the presented with changes in vision and paresthesias. Her initial workup from the neurologist was negative and further workup was delayed until her symptoms worsened. After worsening symptoms, MRI revealed that multiple sclerosis was the cause of her symptoms.
\end{abstract}

Keywords: Multiple Sclerosis; Relapsing Remitting Multiple Sclerosis

\section{Case Presentation}

A 40-year-old African American female with a past medical history of anxiety presented to a family medicine clinic with blurry vision in her left eye and intermittent left arm numbness. Prior to presenting to the clinic, she was seen by optometry, and she reported a normal eye exam. She was prescribed steroid eye drops, which had minimal improvement in her symptoms. She had recently started sertraline $25 \mathrm{mg}$ for anxiety, but she was unsure of whether her symptoms started prior to this. She had a normal physical exam including neurological exam. Initial work up included comprehensive metabolic panel, hemoglobin A1c, and CT scan of the head without contrast and were all normal. She followed up 3 months later for her wellness exam with continued symptoms of intermittent left arm and leg numbness. She was referred to neurology. The neurologist noted that the leg numbness was mostly in the evening and improved with stretching. She had a normal neurological exam at this time. The neurologist diagnosed her with restless leg syndrome advised the patient to stretch. Further testing included a B12 level which was normal and an MRI of the brain without contrast. The MRI showed nonspecific patchy white matter disease. The differential in the radiologist report included early microangiopathic disease, migraines, vasculopathy, prior trauma and demyelination. The report noted that additional workup/follow-up should be based on clinical grounds. The neurologist felt that this finding was nonspecific and did not feel that these findings were related to her symptoms. No further evaluation, treatment were planned by the neurologist. The patient inquired about the findings and was not given was told the same. The patient continued to have symptoms and then reached out to her family medicine doctor and expressed her concerns. At her next family medicine visit, she was referred back to neurology again for further evaluation. At the next neurology visit, her paresthesias seemed to be worsening. She was noted to have increased reflexes in her lower extremities and decreased vibratory sensation in her big toes bilaterally. Otherwise, her exam was normal. An MRI of the cervical and thoracic spine showed scattered cervical cord lesions with subtle cord expansion, but without associated enhancement. The MRI of the thoracic spine showed patchy signal abnormality in the lower thoracic cord at T11 without associated enhancement. These findings were worrisome for a demyelinating process. The patient was referred to a demyelinating specialist. Further workup was significant for positive anti-nuclear antibody, positive speckled. Lumbar puncture was significant for oligoclonal bands (13) and CSF I/Albumin ratio (0.33), IgG index (1.38) establishing the diagnosis of relapsing remitting multiple sclerosis. And she was started on disease modifying therapy. The time from initial presentation to her diagnosis which was 7 months. The patient was initially denied approval for treatment from her insurance company and is still in the process of getting her treatment approved.

\section{Discussion}

Multiple sclerosis can be difficult to diagnose due to non-specific clinical presentations. It tends to be more common in areas in temperate zones in northern Europe, Russia, Canada, and northern states of the USA [1]. Some of the clinical features of multiple sclerosis include repeated attacks separated in time, multiple CNS sites affected, and progressive neurological impairment [1]. Urge incontinence, retro orbital neuritis, diplopia, nystagmus, intranuclear ophthalmoplegia, spastic paraparesis, or spastic-ataxic gait are among other signs and symptoms of multiple sclerosis [1]. Lhermitte's sign is an early sign of multiple sclerosis [1]. Lhermitte's sign is an early sign of multiple sclerosis and occurs when the patient feels an electric shock going down the spine that can be provoked by forceful flexion of the neck [1]. Sensory disturbances are common and occur early in the disease, particularly a decrease in vibratory sensation [1]. Because multiple sclerosis affects multiple CNS sites and the symptoms may be intermittent, it may be difficult to diagnose and the diagnosis may be delayed. The time from initial presentation to diagnosis by a specialist is commonly greater than 5 months [2].

This patient initially presented with blurry vision, which may have been a sign of retro orbital neuritis and intermittent limb numbness. However, she did report a normal eye exam. She was referred to a neurologist within the first few months of presentation. However, even the neurologist did not suspect multiple sclerosis on his initial evaluation. It was the patient's worsening of symptoms that 
prompted further work up leading to her eventual diagnosis. She had nonspecific findings on her brain MRI, and there was not a clear explanation of her symptoms after her initial neurology consultation. In this case, there should have been continued exploration of the patient's symptoms after the initial workup. The timing of detection is important in multiple sclerosis since earlier treatment with disease modifying therapy is associated with decreased disability compared to a delay in therapy [3]. So with that being said, it is important when seeing patients with nonspecific symptoms, to keep your differential diagnosis broad and to continue to continue to investigate the cause of a patient's symptoms if a diagnosis is not clear and the patient is still symptomatic. This patient is has still not started disease modifying treatment due to insurance denial for Mavenclad (cladribine), but she is in the process of having the denial appealed leading to a further delay in her treatment.

\section{References}

1. Mumenthaler M, Mattle H, Taub E. Clinical Syndrome. $4^{\text {th }}$ edn. Thieme. 2003.

2. Fernández $\mathrm{O}$, Fernández $\mathrm{V}$, Arbizu T, Izquierdo G, Bosca I, Arroyo R, et al. Characteristics of multiple sclerosis at onset and delay of diagnosis and treatment in Spain (the Novo Study). J Neurol. 2010; 257: 1500-1507.

3. O'Connor P, Goodman A, Ludwig K, Lublin F, Polman C, Rudick RA, et al.Long-term safety and effectiveness of natalizumab redosing and treatment in the STRATA MS Study. Neurology. 2014; 83: 78-86. 